\title{
O Ranking Brasileiro de Pesquisa e os Princípios de Berlim Para Rankings de Instituições de Ensino Superior
}

\author{
Sibele Fausto \\ Bibliotecária da Universidade de São Paulo (USP), Brasil \\ sifausto@usp.br \\ Clara Calero-Medina \\ Pesquisadora do Centro de Estudos de Ciência e Tecnologia da Universidade de Leiden, \\ Holanda \\ clara@cwts.leidenuniv.nl \\ Ed Noyons \\ Pesquisador do Centro de Estudos de Ciência e Tecnologia da Universidade de Leiden, \\ Holanda \\ noyons@cwts.leidenuniv.nl
}

\section{RESUMO}

Os rankings estão ganhando destaque como instrumentos de avaliação das instituições de ensino superior. Nos últimos anos, surgiram vários deles, tanto nacionais quanto internacionais. O Ranking Brasileiro de Pesquisa (BRR) foi lançado em 2014 no Brasil e mede o desempenho das instituições de pesquisa científica brasileiras. Usando um sofisticado conjunto de indicadores bibliométricos, o ranking tem como objetivo fornecer medições altamente precisas do impacto científico dessas organizações e de seu envolvimento na colaboração científica. Sua fonte de dados é a base Web of Science, considerando publicações indexadas no período entre 2003 e 2012. No presente artigo, é analisado se o BRR segue as recomendações do documento "Princípios de Berlim para Rankings de Instituições de Ensino Superior", elaborado em 2006 pelo Grupo Internacional de Especialistas em Rankings, que contém um conjunto de 16 diretrizes para orientar os produtores de rankings na elaboração de suas classificações. O cotejo das características do BRR com os Princípios de Berlim mostrou que esse ranking está perto de completar sua conformidade aos princípios recomendados para rankings. 


\title{
FutureJournal
}

PALAVRAS-CHAVE: Rankings. Ranking Brasileiro de Pesquisa. Diretrizes. Princípios de Berlim para Rankings de Instituições de Ensino Superior.

\section{The Brazilian Research Ranking and the Berlin Principles for Rankings of Higher Education Institutions}

\begin{abstract}
University rankings are gaining prominence as assessment tools of higher education institutions. In recent years there have emerged various rankings, either with national or international focus. The CWTS Brazilian Research Ranking (BRR) was launched in 2014 in Brazil and measures the performance of Brazilian scientific research institutions (not just universities). Using a sophisticated set of bibliometric indicators, this ranking aims to provide highly accurate measurements of the scientific impact of these organizations and their involvement in scientific collaboration, and its data source is the Web of Science database, considering indexed publications between 2003 and 2012. The aim of this paper is an analysis and a discussion if the BRR follows the recommendations from the document "Berlin Principles for Higher Education Institutions Rankings", published in 2006 by the International Rankings Expert Group, which contains a set of sixteen guidelines to guide producers in developing their rankings. The comparison of the BRR with the Berlin principles showed that this ranking is close to complete its accordance with the recommendations for rankings.
\end{abstract}

KEY-WORDS: Rankings. Brazilian Research Ranking. Guidelines. Berlin Principles for Rankings of Higher Education Institutions. 


\section{INTRODUÇÃO}

O ensino superior atualmente experimenta um processo de massificação, mercantilização e globalização (Shin, Toutkoushian \& Teichler, 2011). De fato, recente relatório publicado pela Organização das Nações Unidas para a Educação, a Ciência e a Cultura - United Nations Educational, Scientific and Cultural Organization - (Unesco, 2015) indica as tendências e os desenvolvimentos em ciência, tecnologia, política de inovação e governança no período entre 2009 e meados de 2015, mostrando um contínuo crescimento do ensino superior em nível mundial: o número de estudantes internacionais aumentou de 2,8 milhões para 4,1 milhões entre 2005 e 2013. Segundo o relatório, a partir de 2012 cinco países tinham mais de 10.000 de seus estudantes de doutorado no exterior: China (58.492 estudantes), Índia (30.291), Alemanha (13.606), Irã (12.180) e República da Coreia (11.925). Dez outros tiveram mais de 4.000 (Itália, Canadá, EUA, Arábia Saudita, Indonésia, França, Vietnã, Turquia, Paquistão e Brasil), projetando um forte impulso na globalização da educação superior mundial. Na Figura 1, mostra-se que essa evolução do crescimento da internacionalização do ensino superior é contínua desde 1975.

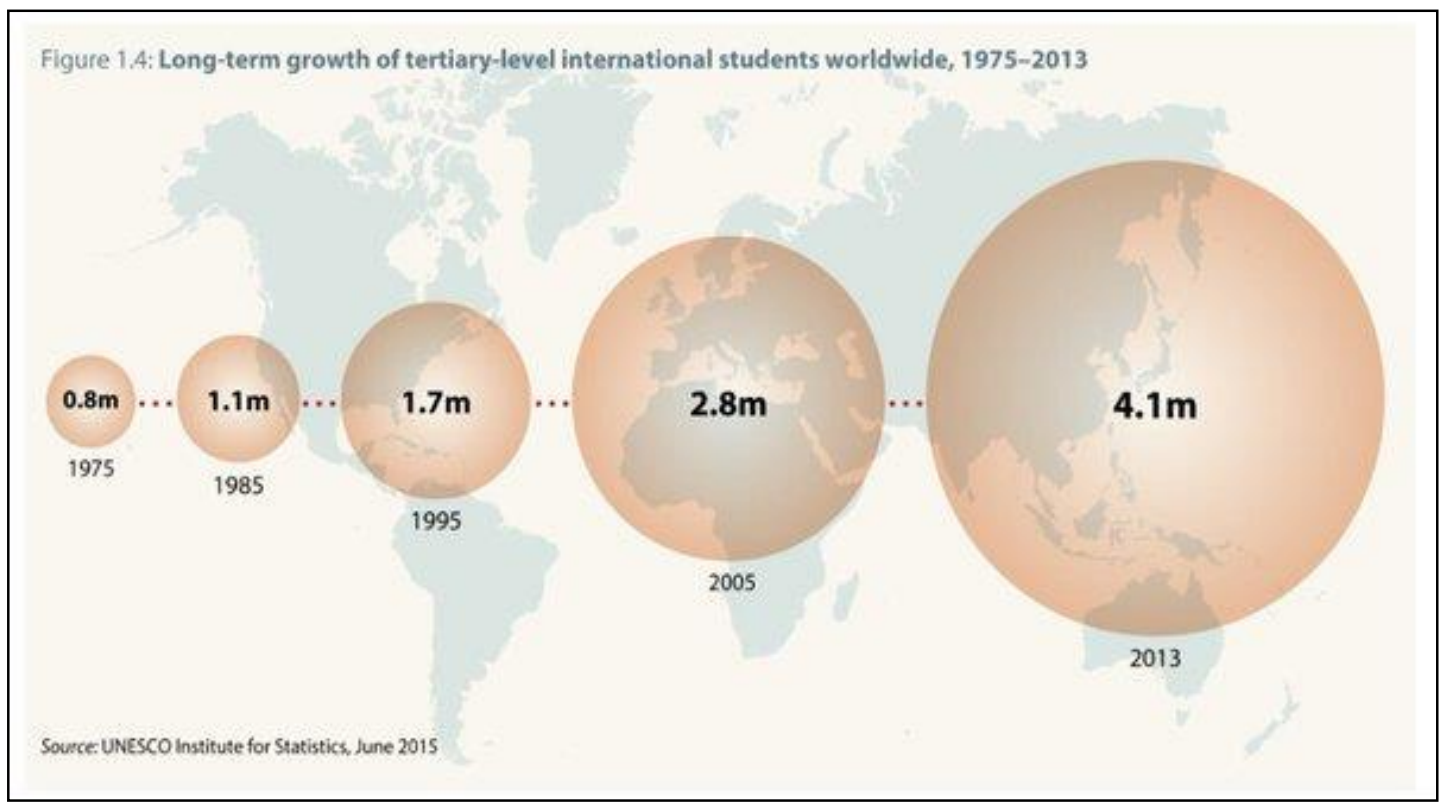

Figura 1: Crescimento mundial no longo prazo de estudantes internacionais do nível superior (1975-2013)

Fonte: Unesco (2015) 
Nesse cenário, os rankings universitários ganham destaque como instrumentos de avaliação e classificação das universidades, principalmente após o lançamento, em 2003, do primeiro ranking em âmbito mundial, o Academic Ranking of World Universities (ARWU), também conhecido como Ranking de Shanghai, lançado pela Universidade Shanghai Jiao Tong, da China. Na sequência, surgiram outros rankings mundiais, tais como o Times Higher Education World University Rankings (THE) e o Webometrics Rankings (WR), ambos lançados em 2004; o CWTS Leiden Ranking em 2007; o SCImago Institutions Rankings World Report (SIR) em 2009; e o Quacquarelli Symonds World University Rankings (QS) em 2010, entre outros. Esses sistemas ganham crescente importância atual na sociedade, como sinalizadores de um paradigma de excelência e qualidade de instituições de ensino superior (IES) no mundo globalizado.

Porém, rankings de universidades já existiam antes das atuais versões mundiais. O primeiro ranking universitário em âmbito nacional, o America's Best Colleges, foi publicado nos Estados Unidos em 1983 pelo semanário U.S. News \& World Report (Webster, 2001), também seguido de outros com escopo nacional, criados com o objetivo primário de orientar os estudantes estadunidenses na escolha da universidade para a continuação de seus estudos após o exame Scholastic Aptitude Test ou Scholastic Assessment Test (SAT) - um instrumento de admissão ao ensino superior dos EUA que permite ao estudante a escolha entre várias opções de universidades, simultaneamente (Almeida Filho, 2011).

A experiência estadunidense inspirou a criação de sistemas nacionais de classificação de universidades por outros países. Usher e Medow (2009) analisaram 22 rankings nacionais de 15 países diferentes (Austrália, Canadá, Cazaquistão, Chile, China, Espanha, Estados Unidos, Holanda, Hong Kong, Itália, Peru, Polônia, Reino Unido, Taiwan e Ucrânia), apontando sua proliferação. O Brasil já conta com dois rankings universitários em nível nacional: o Ranking Universitário da Folha (RUF, 2015), lançado pelo jornal Folha de S.Paulo em 2012 e já na sua quinta edição; e o Ranking Brasileiro de Pesquisa (CWTS Brazilian Research Ranking - BRR (2015), lançado em maio de 2014 e elaborado pelo Centro para Estudos de Ciência e Tecnologia de Leiden (Centre for Science and 
Technology Studies, ou Centrum voor Wetenschap en Technologische Studies - CWTS), instituição holandesa dedicada aos estudos e métricas de Ciência e Tecnologia (C\&T), ligada à Universidade de Leiden.

Tal expansão de rankings nacionais pode ser interpretada como uma reação às classificações globais por países cujas IES neles são desconsideradas, ou mesmo para complementar aspectos nacionais e locais que não figuram entre os critérios dessas listagens internacionais.

Com a crescente importância do fenômeno dos rankings, evidenciando sua influência em decisões individuais no momento de optar por uma universidade, na reputação de instituições ranqueadas e em políticas voltadas ao ensino superior - não sem polêmicas desencadeadas sobre as metodologias adotadas pelas diversas classificações -, surge o entendimento de que tais aparatos devem ser acompanhados, garantindo a devida atenção aos procedimentos éticos. Assim, a Organização das Nações Unidas para a Educação, a Ciência e a Cultura (United Nations Educational, Scientific and Cultural Organization - Unesco), em parceria com órgãos internacionais para a educação, cria em 2004 o Grupo Internacional de Especialistas em Rankings (International Ranking Expert Group - IREG), que em 2006 elabora o documento "Princípios de Berlim para Rankings de Instituições de Ensino Superior", um conjunto de 16 princípios recomendados para nortear os produtores de rankings na qualidade e nas boas práticas na elaboração de suas classificações de IES. Neste trabalho, analisa-se o Ranking Brasileiro de Pesquisa (BRR), com o objetivo de verificar sua conformidade aos Princípios de Berlim.

\section{METODOLOGIA}

Para alcançar o objetivo proposto, procede-se a uma breve explanação sobre os critérios considerados pelos rankings ao classificar universidades. A seguir, as características do BRR são descritas, bem como as diretrizes dos Princípios de Berlim para rankings de instituições de ensino superior. As 16 diretrizes do documento são então cotejadas com as informações disponíveis do BRR, acompanhadas por análise e discussão, atribuindo-se uma pontuação de conformidade a cada um dos princípios de 
Berlim, por meio de uma adaptação da Escala de Likert (Badri, Donald \& Donna, 1995), com cinco scores que variam de "Não se aplica" (NA), significando que o Princípio de Berlim em questão não é aplicável ao BRR, não valendo pontuação; "Não atende", em que o BRR não cumpre com o requisito do princípio, com valor de $0 \%$; "Atende parcialmente", significando que existe alguma adesão do BRR ao princípio relacionado, valendo $50 \%$; "Atende", em que o requisito do princípio é atendido pelo $B R R$, valendo 75\%; e "Atende plenamente", quando o requisito do princípio é atendido e superado, com valor de $100 \%$. Por fim, os resultados são consolidados para verificação do nível de conformidade do BRR aos Princípios de Berlim para Rankings de Instituições de Ensino Superior.

\section{OS CRITÉRIOS CONSIDERADOS PELOS RANKINGS DE UNIVERSIDADES}

Segundo Shin (2011), os critérios considerados pelos rankings de universidades são relacionados a dimensões da eficácia institucional representadas em algumas medidas de indicadores de ambiente acadêmico (campus life), ensino (teaching), produção de pesquisa (research) e reputação (reputation). Há rankings que, além dessas dimensões básicas, consideram outras dimensões como a internacionalização das IES - dada a importância da globalização e o intenso movimento transnacional de pessoal acadêmico (estudantes e pesquisadores); a inovação, caracterizada como a interface da universidade com a indústria e pelo registro de patentes; e a presença na Web, reconhecendo que o meio virtual tem papel cada vez mais importante na disseminação do conhecimento gerado pelas universidades.

Os dados obtidos para ranquear as universidades podem ser internos às instituições - fornecidos pelas próprias universidades; ou externos - extraídos de bases de dados como a Web of Science (WoS) e a Scopus, para auferir o número de publicações e o número de citações atribuídas às instituições; e/ou através de pesquisas de opinião junto a stakeholders, por exemplo. Verifica-se que na maioria das atuais classificações de universidades os critérios mais presentes são aqueles 
relacionados à dimensão da pesquisa (research), valorizando aspectos da produção científica e tecnológica das instituições.

Para Shin e Toutkoushian (2011), é muito difícil estabelecer indicadores de certas dimensões institucionais tais como a qualidade do ensino (teaching quality), fazendo com que a maioria dos rankings universitários, especialmente os globais, dependa fortemente de medidas quantificáveis de desempenho institucional. Shin (2011) indica ainda que os rankings internacionais não consideram dimensões locais da efetividade das IES, pois tais aspectos configuram uma medida difícil de captar (principalmente em nível mundial), devido às variabilidades das comunidades no entorno das universidades. Nesse sentido, os rankings nacionais ganham importância ao situarem um contexto local de produção e impacto da atuação das universidades, que são desconsiderados em rankings globais. Sanz-Casado, Garcia-Zorita, Serrano Lopez, Efraín-Garcia e De Filippo (2013) apontam que os rankings nacionais possuem características que os tornam mais apropriados para identificar e comparar atividades das IES de uma mesma região e país.

\section{O RANKING BRASILEIRO DE PESQUISA (CWTS BRAZILIAN RESEARCH RANKING - BRR)}

O BRR é baseado na metodologia do global CWTS Leiden Ranking, e como este também se abstém de combinar arbitrariamente múltiplas dimensões do desempenho da universidade em um único indicador agregado. O BRR também não se baseia em dados fornecidos pelas próprias universidades, tampouco se utiliza de dados de pesquisa de opinião (Waltman et al., 2012), comuns a muitos rankings. Assim, o BRR não avalia dimensões institucionais como reputação, ambiente de ensino, etc., e classifica as instituições brasileiras em um conjunto de indicadores bibliométricos relacionados à produção científica organizada em duas dimensões: Impacto e Colaboração. As instituições de ensino superior brasileiras são classificadas em redes públicas, privadas e especiais, de acordo com a classificação brasileira do Ministério da Educação e Cultura (MEC). Além de universidades, o BRR inclui institutos de investigação, hospitais e organizações de economia mista (a exemplo da Petrobras). Seus 
indicadores são tanto dependentes como independentes do tamanho das instituições, incluindo intervalos de estabilidade para as medidas independentes do tamanho (CWTS Brazilian Research Ranking, 2015).

A dimensão do Impacto considera os seguintes indicadores: Produção total $(P)$, que se refere ao total da produção publicada das instituições indexada na WoS sob os critérios considerados pelo ranking; Quantidade de publicações nas revistas consideradas Top entre as $10 \%$ mais citadas $\left(\mathrm{P}_{\text {Top } 10 \%}\right)$; Proporção de $10 \%$ de publicações consideradas Top (Publication Proportion - $\mathrm{PP}_{\text {Top } 10 \%)}$, que se refere à proporção de publicações de uma universidade que pertence às revistas entre as $10 \%$ mais citadas, em comparação com outras publicações no mesmo campo e no mesmo ano; Média de Pontuação da Citação (Mean Citation Score - MCS), que é o número médio de citações das publicações de uma universidade; e a Média de Pontuação da Citação Normalizada (Mean Normalized Citation Score MNCS), que é o número médio de citações das publicações de uma universidade, normalizada para as diferenças de campo e ano de publicação.

Já a dimensão Colaboração Científica considera os seguintes indicadores: Proporção de publicações interinstitucionais em colaboração $\left(\mathrm{PP}_{\text {collab }}\right)$, relacionado à proporção de publicações de uma universidade em coautoria com outras organizações; Proporção de publicações em

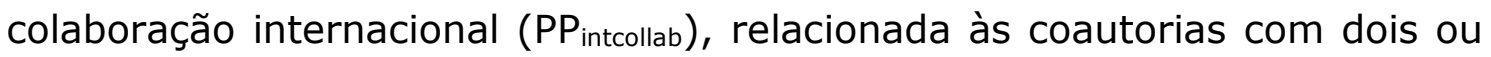
mais países; Proporção de publicações de colaboração da Universidade com a Indústria ( $\left(P_{\text {collabu-I }}\right)$, que refere-se às coautorias com um ou mais parceiros industriais; além de considerar indicadores de distância das colaborações (Mean Geographical Collaboration Distance - MGCD), com a Proporção de publicações colaborativas de curta distância $\left(P_{<100} \mathrm{~km}\right)$, considerando as publicações de uma universidade com uma distância geográfica de colaboração menor que 100 km; e a Proporção de publicações colaborativas de longa distância $\left(P_{>1000} \mathrm{~km}\right)$, que é a proporção de publicações de uma universidade com uma distância geográfica de colaboração maior que $1000 \mathrm{~km}$. O BRR, assim como o CWTS Leiden Ranking, não pondera seus critérios, dada a sofisticada agregação 
estatística derivada dos indicadores MCS, MNCS e MGCD. No Quadro 1, sumarizam-se as dimensões e os indicadores considerados pelo BRR.

\begin{tabular}{|c|c|}
\hline Dimensão & Indicadores \\
\hline Impacto & $\begin{array}{l}\text { P - Produção total } \\
\text { PTop } 10 \% \text { - Quantidade de publicação entre as Top }_{10 \%} \\
\text { PP }_{\text {Top } 10 \%} \text { - Proporção de } 10 \% \text { de publicações Top } \\
\text { MCS - Média de Pontuação da Citação } \\
\text { MNCS - Média de Pontuação da Citação } \\
\text { Normalizada }\end{array}$ \\
\hline Colaboração & 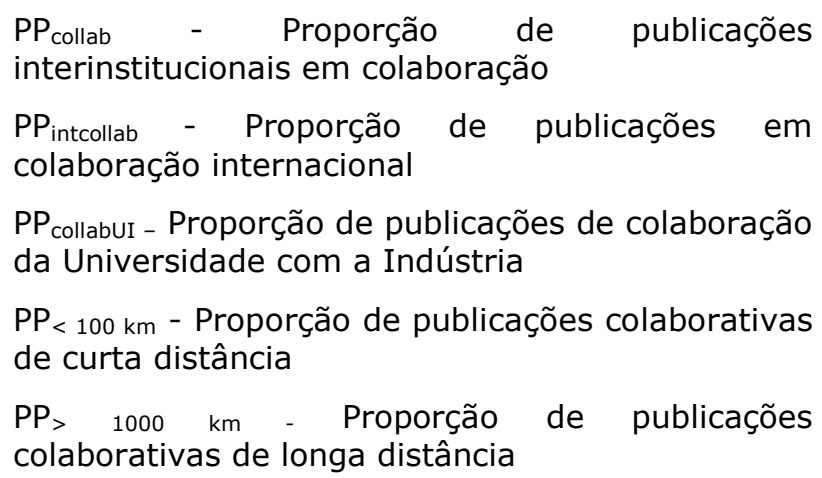 \\
\hline
\end{tabular}

Quadro 1: Dimensões e indicadores do BRR

Fonte: CWTS Brazilian Research Ranking, 2015

\section{OS PRINCÍPIOS DE BERLIM SOBRE RANKINGS DE INSTITUIÇÕES DE ENSINO SUPERIOR}

A proliferação dos rankings de universidades foi acompanhada por extensas e variadas controvérsias relacionadas a suas metodologias (Teichler, 2011). Tal situação levou a Unesco, através de seu órgão subordinado Centro Europeu para o Ensino Superior (Centre Européen pour I'Enseignement Supérieur - CEPES), a tomar a iniciativa de reunir, em 2004, consultores ad-hoc e entidades colaboradoras, como a Associação Universitária Europeia (European University Association - EUA), o Instituto de Política de Educação Superior (Institute for Higher Education Policy), de Washington, DC, e o Centro de Desenvolvimento do Ensino Superior (Centrum für Hochschulentwicklung - CHE) alemão, resultando na criação do Grupo Internacional de Especialistas em Rankings (International Ranking Expert Group - IREG). Em 20 de maio de 2006, o IREG apresenta o 
documento "Princípios de Berlim para Rankings de Instituições de Ensino Superior" (IREG, 2006) - um conjunto de 16 diretrizes para orientar os produtores de rankings na elaboração de suas classificações com base em quatro categorias:
i. objetivos e metas dos rankings;
ii. metodologia adotada: escolha e peso dos indicadores;
iii. coleta e processamento de informações;
iv. apresentação dos resultados no ranking.

As categorias dos Princípios de Berlim são detalhadas no Quadro 2.

\begin{tabular}{|c|c|}
\hline Categorias & Princípios \\
\hline $\begin{array}{lr}\text { Objetivos } & \text { e } \\
\text { Metas } & \text { dos } \\
\text { Rankings } & \end{array}$ & $\begin{array}{l}\text { 1. Ser uma entre várias diferentes abordagens para a avaliação } \\
\text { do ensino superior. Rankings podem fornecer informações } \\
\text { comparativas e melhor compreensão do ensino superior, mas } \\
\text { não devem ser o principal método para avaliar uma instituição } \\
\text { de ensino superior. Rankings fornecem uma perspectiva } \\
\text { baseada no mercado que pode complementar o trabalho do } \\
\text { governo, das instituições de acreditação e das agências de } \\
\text { avaliação independentes. } \\
\text { 2. Ser claros quanto ao propósito e ao público ao qual estão } \\
\text { dirigidos. Os indicadores utilizados para atender a um objetivo } \\
\text { específico ou para informar um público-alvo podem não ser } \\
\text { adequados para diferentes fins, ou grupos-alvo. } \\
\text { 3. Reconhecer a diversidade das instituições e levar em conta } \\
\text { diferentes missões e objetivos. Medidas de qualidade para as } \\
\text { instituições voltadas para a pesquisa, por exemplo, são } \\
\text { bastante diferentes daquelas apropriadas para as instituições } \\
\text { que oferecem amplo acesso às comunidades carentes. As } \\
\text { instituições que estão sendo classificadas e os peritos que } \\
\text { assistem ao processo de classificação devem ser consultados } \\
\text { frequentemente. } \\
\text { 4. Ser claros sobre as fontes de informação e seu significado. A } \\
\text { relevância dos resultados de classificação depende do público } \\
\text { que recebe as informações e das fontes dessas informações } \\
\text { (tais como bases de dados, alunos, professores, entidades } \\
\text { empregadoras). Uma boa prática seria combinar diferentes } \\
\text { perspectivas fornecidas por essas fontes, a fim de obter uma } \\
\text { visão mais completa de cada instituição de ensino superior } \\
\text { incluída no ranking. } \\
\text { 5. Especificar os contextos linguístico, cultural, econômico e } \\
\text { histórico do sistema avaliado. Rankings internacionais, em } \\
\text { particular, devem estar atentos à possibilidade de viés e ser } \\
\text { precisos na determinação de seu objetivo. Nem todas as } \\
\text { nações ou sistemas compartilham os mesmos valores e crenças } \\
\text { sobre o que constitui a "qualidade" em instituições de ensino } \\
\text { superior, e os sistemas de classificação não devem ser } \\
\text { concebidos para forçar essas comparações. }\end{array}$ \\
\hline
\end{tabular}

Continua 


\section{Categorias \\ Princípios}

Metodologia

adotada

6. Deve haver transparência da metodologia. A escolha dos métodos usados para preparar rankings deve ser clara e inequívoca. Essa transparência deve incluir o cálculo dos indicadores, bem como a origem dos dados.

7. Escolha de indicadores de acordo com sua relevância e validade. A escolha dos dados deve ser baseada no reconhecimento da capacidade de cada medida para representar a qualidade acadêmica e as forças institucionais, e não na disponibilidade de dados. Deve haver clareza sobre o porquê de as medidas terem sido incluídas e o que elas pretendem representar.

8. Preferência por mensurar resultados. Os dados sobre os insumos são relevantes, pois refletem a condição geral de determinado estabelecimento e são mais frequentemente disponíveis. Medidas de resultados fornecem uma avaliação mais precisa da posição e/ou a qualidade de determinada instituição ou programa. Os compiladores de rankings devem garantir que um equilíbrio adequado seja alcançado.

9. Destaque para os pesos atribuídos aos indicadores (se usados) e limitação das mudanças feitas neles. Mudanças nos pesos tornam difícil para os consumidores entender se a posição do programa ou da instituição mudou no ranking devido a uma diferença inerente ou devido a uma mudança metodológica.

Coleta processamento de informações e 10. Devem-se respeitar os padrões éticos e as recomendações de boas práticas destes Princípios. A fim de assegurar a credibilidade de cada ranking, os responsáveis pela coleta e uso de dados e realização de visitas in loco devem ser os mais objetivos e imparciais possíveis.

11. Usar informações auditáveis e verificáveis sempre que possível. Tais dados têm várias vantagens, incluindo o fato de que foram aceites por instituições e que são comparáveis e compatíveis entre as instituições.

12. Incluir informações obtidas conforme os procedimentos adequados à coleta científica de dados. Os dados coletados a partir de um subconjunto não representativo ou distorcido de estudantes, professores ou outras partes podem não representar uma instituição ou programa e devem ser excluídos.

13. Aplicar medidas de garantia de qualidade aos processos do próprio ranking. Os processos utilizados para avaliar as instituições devem ser usados para avaliar o próprio ranking. Rankings devem ser continuamente sistematizados para desenvolver uma melhor metodologia.

14. Aplicar medidas organizacionais para aprimorar a credibilidade. Essas medidas podem incluir órgãos consultivos ou mesmo de supervisão, de preferência com alguma participação internacional. 


\begin{tabular}{|l|l|l|}
\hline \multicolumn{1}{|c|}{ Categorias } & \multicolumn{1}{|c}{ Continuação } \\
\hline $\begin{array}{l}\text { Apresentação } \\
\text { dos resultados } \\
\text { no ranking }\end{array}$ & $\begin{array}{l}\text { O ranking deve proporcionar aos consumidores uma } \\
\text { compreensão clara de todos os fatores usados em sua } \\
\text { elaboração e oferecer escolhas na forma de apresentação. } \\
\text { Dessa forma, os usuários dos rankings terão melhor } \\
\text { compreensão dos indicadores utilizados para classificar as } \\
\text { instituições ou programas. Além disso, devem ter a } \\
\text { oportunidade de tomar as próprias decisões sobre como esses } \\
\text { indicadores devem ser ponderados. } \\
16 . \text { Ser compilado de modo a eliminar ou reduzir erros nos } \\
\text { dados originais e ser organizado e apresentado de forma tal } \\
\text { que erros e falhas possam ser corrigidos. As instituições e o } \\
\text { público devem ser informados sobre os erros que ocorreram. }\end{array}$ \\
\hline
\end{tabular}

\section{Quadro 2: Princípios de Berlim para rankings de Instituições de Ensino Superior}

Fonte: IREG (2006)

\section{Resultados: O BRR e os Princípios de Berlim}

As 16 diretrizes dos Princípios de Berlim sobre Rankings de Instituições de Ensino Superior foram cotejadas com as informações disponíveis do BRR, acompanhadas por análise e discussão de acordo com as categorias do documento do IREG, sendo atribuído um score à conformidade do BRR ao requisito do princípio, conforme indicado na Metodologia: "Não se aplica" (NA); "Não atende", com valor de 0\%; "Atende parcialmente", valendo 50\%; "Atende", com valor de 75\%; e "Atende plenamente", com valor de $100 \%$.

\subsection{CATEGORIA: PROPÓSITOS E OBJETIVOS}

\subsubsection{Princípio 1}

Ser uma entre várias diferentes abordagens para a avaliação do ensino superior (IREG, 2006).

O BRR não é a única maneira pela qual as instituições de ensino superior brasileiras são avaliadas, existindo outros instrumentos com essa finalidade, tanto governamentais quanto privados. No âmbito 
governamental, as avaliações são realizadas trienalmente pelo Instituto Nacional de Estudos e Pesquisas Educacionais Anísio Teixeira (Inep), órgão do MEC. As avaliações são fundamentadas na Lei no 10.861, de 2004, que instituiu o Sistema Nacional de Avaliação da Educação Superior (Sinaes); e na Portaria Normativa no 40, de 2007, Art. 33-B, que institui o e-MEC, sistema eletrônico de fluxo de trabalho e gerenciamento de informações relativas aos processos de regulação, avaliação e supervisão da educação superior. Os indicadores do Inep baseiam-se em três parâmetros: I - de cursos superiores: através do Conceito Preliminar de Curso (CPC); II - de instituições de educação superior: pelo Índice Geral de Cursos Avaliados da Instituição (IGC); e III - de desempenho de estudantes: pelo conceito obtido a partir dos resultados do Exame Nacional de Desempenho de Estudantes (Enade). Além disso, há também a avaliação dos cursos de pósgraduação no país pela Coordenação de Aperfeiçoamento de Pessoal de Nível Superior (Capes).

Outro instrumento, dessa vez privado, de avaliação das IES brasileiras é o Ranking Universitário da Folha (RUF), lançado pelo jornal Folha de S.Paulo em 2012 e atualmente na sua quinta edição. O RUF classifica as IES brasileiras a partir de dois focos: o ranking de universidades e o ranking de cursos. A versão relacionada às universidades considera 195 universidades públicas e privadas avaliadas por meio de cinco dimensões com pesos específicos (Folha de S.Paulo, 2016).

BRR Score: Atende (75\%).

\subsubsection{Princípio 2}

Ser claros quanto ao propósito e ao público ao qual estão dirigidos (IREG, 2006).

Na página de abertura de seu site oficial, o BRR indica de forma explícita seu objetivo: "Fornecer medições altamente precisas do impacto principalmente científico destas organizações e do seu envolvimento na colaboração científica" (CWTS Brazilian Research Ranking, 2015). Na mesma página inicial, o BRR também indica o público-alvo do ranking todos os atores envolvidos com a avaliação da pesquisa brasileira: 
Brazil is an important fast growing country in science. As a result research evaluation is gaining importance. Bibliometric indicators can be supportive in that process. With this initiative CWTS wishes to contribute to this task providing methodology as well as results based on many years of experience in this area (CWTS Brazilian Research Ranking, 2015).

BRR Score: Atende (75\%).

\subsubsection{Princípio 3}

Reconhecer a diversidade das instituições e levar em conta diferentes missões e objetivos (IREG, 2006).

Segundo Vogel, Milanez, Noyons, Kobashi \& Faria (2014), o CWTS Leiden contou com a colaboração de dois pesquisadores brasileiros em sua equipe de elaboração do BRR, no processo de normalização de nomes, na classificação das organizações e em outros aspectos do ranking. O BRR considera a diversidade das instituições de acordo com a categorização do próprio MEC, classificando universidades públicas, privadas e especiais e institutos de pesquisa, estabelecimentos hospitalares em geral e universitários e organizações de capital misto (a exemplo da Petrobras) (CWTS Brazilian Research Ranking, 2015).

BRR Score: Atende (75\%).

\subsubsection{Princípio 4}

Ser claros sobre as fontes de informação e seu significado (IREG, 2006).

O BRR 2014 indica que sua fonte é a base de dados Web of Science (WoS) da empresa Thomson Reuters, localizada na Filadélfia, EUA; e considerando publicações indexadas entre 2003 e 2012 (CWTS Brazilian Research Ranking, 2015). A WoS reúne as antigas bases do Institute for Scientific Information (ISI) depois de sua aquisição pela Thomson Reuters nos anos 1990: Science Citation Index Expanded (SCI), Social Sciences Citation Index (SSCI) e Arts \& Humanities Citation Index (AHCI). Em setembro de 2014, a WoS apresentava 90 milhões de registros, incluindo mais de um bilhão de referências citadas (Thomson Reuters, 2014). A WoS 
é considerada uma base de dados prestigiada (Cavacini, 2015), mas há críticas por sua restrição de indexação de títulos de revistas e de áreas de pesquisa (Hood \& Wilson, 2001).

BRR Score: Atende (75\%).

\subsubsection{Princípio 5}

Especificar os contextos linguístico, cultural, econômico e histórico do sistema avaliado (IREG, 2006).

Este princípio assemelha-se ao 30 anteriormente citado, no reconhecimento da diversidade das instituições, com seus diferentes contextos. O BRR é baseado na metodologia do global CWTS Leiden Ranking, que mesmo em âmbito mundial prevê a análise de diferentes instituições por meio de suas características e dimensões semelhantes, de acordo com seu arranjo no país de origem (Vogel et al., 2014). Essa forma de avaliar aproxima instituições parecidas umas com as outras em termos de missão e tipologia institucional, sendo mais judiciosa em relação à diversidade de instituições e sistemas existentes (instituições públicas e privadas, organizações de pesquisa - mesmo aquelas de capital misto, e hospitais universitários), além de classificar os componentes, grupos e afiliações, em sete diferentes áreas do conhecimento, considerando tal diversidade.

BRR Score: Atende (75\%).

\subsection{CATEGORIA: METODOLOGIAS}

\subsubsection{Princípio 6}

Deve haver transparência da metodologia (IREG, 2006).

A metodologia do BRR é disponibilizada publicamente no site do ranking (CWTS Brazilian Research Ranking, 2015), no idioma inglês. No idioma português, há o trabalho de Vogel et al. (2014) também documentando a metodologia do BRR. E como já indicado, essa metodologia é baseada no CWTS Leiden Ranking - também já documentada por 
Waltman et al. (2012). A extensa discussão metodológica que precedeu o lançamento do CWTS Leiden Ranking igualmente foi documentada por Waltman et al. (2011a, 2011b) e Waltman e Schereiber (2013).

BRR Score: Atende plenamente (100\%).

\subsubsection{Princípio 7}

Escolha de indicadores de acordo com sua relevância e validade (IREG, 2006).

Como já indicado no princípio 6 anterior, a metodologia do BRR, baseada no CWTS Leiden Ranking, é fundamentada em extenso trabalho de discussão e análise metodológica - incluindo o cálculo dos indicadores e estabelecendo sua relevância e validade -, empreendida pelos pesquisadores ligados ao Centro de Estudos de Ciência e Tecnologia de Leiden (CWTS Leiden), da Universidade de Leiden, Holanda (Waltman et al., 2011a, 2011b; Waltman et al., 2012; Waltman \& Schreiber, 2013).

O BRR adota sofisticadas mensurações estatísticas considerando contagem completa (full counting); contagem fracionada (fractional counting); contagem com dependência ou independência do tamanho da instituição; além de atribuir um intervalo de idade dos dados por meio da técnica estatística conhecida como bootstrapping, que distribui mais equitativamente o peso de publicações colaborativas; e também normaliza os pesos dos indicadores de impacto por área - o BRR considera sete diferentes áreas para sua classificação. Todas essas medições tornam possível a comparação de diferentes instituições em diferentes campos (CWTS Brazilian Research Ranking, 2015).

BRR Score: Atende plenamente (100\%).

\subsubsection{Princípio 8}

Preferência por mensurar resultados (IREG, 2006).

O BRR é explícito em seus objetivos: "Fornecer medições altamente precisas do impacto principalmente científico destas organizações e do seu envolvimento na colaboração científica" (CWTS Brazilian Research Ranking, 
2015). O BRR avalia aspectos mais específicos das instituições, relacionados com seu desempenho científico: seu resultado na produção e na colaboração científica.

BRR Score: Atende plenamente (100\%).

\subsubsection{Princípio 9}

Destaque para os pesos atribuídos aos indicadores (se usados) e limitação das mudanças feitas neles (IREG, 2006).

Este princípio relaciona-se a diversas críticas recebidas pelos rankings devido às variabilidades existentes nas ponderações, de forma nem sempre clara (Buela-Casal, Gutiérrez-Martinez, Bermúdez-Sánchez \& Vadillo-Muñoz, 2007; ). O BRR, tal como o CWTS Leiden Ranking, não atribui pesos a seus indicadores, pois, conforme já indicado, adota um conjunto de sofisticadas análises estatísticas que prescinde de hierarquia determinada por pesos atribuídos. O próprio cálculo estatístico empreendido pelo ranking - considerando a contagem completa (full counting), a contagem fracionada (fractional counting), a contagem com dependência ou independência do tamanho da organização (na opção de parâmetros avançada do ranking), além do intervalo de estabilidade dos dados - já posiciona as instituições de acordo com a característica escolhida para se avaliar (CWTS Brazilian Research Ranking, 2015).

BRR Score: Não se Aplica (NA).

\subsubsection{Princípio 10}

Devem-se respeitar os padrões éticos e as recomendações de boas práticas destes Princípios (IREG, 2006).

O esforço do BRR para alcançar uma metodologia a mais objetiva e imparcial possível para a avaliação das organizações de ensino superior e de pesquisa brasileiras, tal como está implícito em seus objetivos (CWTS Brazilian Research Ranking, 2015), de forma transparente e documentada, está alinhado às boas práticas recomendadas e aos padrões éticos indicados pelos Princípios de Berlim para Rankings. 
BRR Score: Atende (75\%).

\subsection{CATEGORIA: COLETA E TRATAMENTO DE DADOS}

\subsubsection{Princípio 11}

Usar informações auditáveis e verificáveis sempre que possível (IREG, 2006).

Como já indicado, a origem dos dados utilizados pelo BRR para avaliar as instituições brasileiras é a bem estabelecida base de dados WoS, da Thomson Reuters. É uma fonte externa às instituições e, embora seja uma fonte de dados restrita, no Brasil é acessível através do Portal de Periódicos da Capes (2015) por instituições cadastradas, o que permite seu acesso a qualquer tempo para a devida verificação dos dados ali coletados. No entanto, o BRR ressalta em seu site que a atribuição de publicações para organizações de pesquisa não está livre de erros, devido a inconsistências (embora mínimas) inerentes à própria fonte de coleta em relação ao registro dos endereços institucionais na base de dados. A confiabilidade da base de dados é diretamente proporcional à confiabilidade dos resultados do ranking.

BRR Score: Atende (75\%).

\subsubsection{Princípio 12}

Incluir informações obtidas conforme os procedimentos adequados à coleta científica de dados (IREG, 2006).

Conforme já indicado, a fonte de coleta de dados do BRR é a base de dados WoS, considerando todo o conjunto de bases que a compõem: Science Citation Index Expanded (SCI), Social Sciences Citation Index (SSCI) e Arts \& Humanities Citation Index (AHCI). A coleta de dados baseou-se nas publicações do tipo artigo (article) e artigo de revisão (review) com origem institucional do Brasil (através do endereço de afiliação indicado nas publicações), indexadas na WoS durante o período de 2003 a 2012; e considerando apenas o núcleo (core) dessas publicações: 
caracterizadas por seu escopo internacional, por publicarem em inglês e por apresentarem um volume de referências suficientes em relação ao núcleo de periódicos da base WoS. Esses requisitos excluem cerca de $16 \%$ dos periódicos, principalmente aqueles da área de ciências humanas, periódicos comerciais e revistas populares (Vogel et al., 2014; CWTS Brazilian Research Ranking, 2015).

BRR Score: Atende plenamente (100\%).

\subsubsection{Princípio 13}

Aplicar medidas de garantia de qualidade aos processos do próprio ranking (IREG, 2006).

A qualidade dos processos do BRR é indicada pela descrição pormenorizada de sua metodologia, seja na coleta dos dados, seja na identificação das instituições avaliadas, seja nos indicadores adotados, sistematizados pelo CWTS Leiden no objetivo de desenvolver uma metodologia válida (Waltman et al., 2012; CWTS Brazilian Research Ranking, 2015).

BRR Score: Atende plenamente (100\%).

\subsubsection{Princípio 14}

Aplicar medidas organizacionais para aprimorar a credibilidade (IREG, 2006).

O BRR é um produto do CWTS Leiden, que adota princípios e regras fundamentados em rigorosa metodologia científica na sistematização de seus produtos e serviços, que são focados em três eixos: Monitoramento e Avaliação (Monitoring \& Evaluation), com estudos, indicadores e dados (a exemplo dos rankings CWTS Leiden Ranking e BRR, e relatórios e análises encomendados por instituições governamentais e outros públicos); Análise Avançada (Advanced Analytics) por meio de aplicações e ferramentas desenvolvidas pela instituição e disponibilizadas ao público (a exemplo da ferramenta de visualização VOS Viewer, desenvolvida pela instituição; e 
Treinamento e Educação (Training \& Education), oferecendo ao público cursos em análise bibliométrica para a gestão e avaliação da pesquisa.

Deve-se mencionar que tanto para o global CWTS Leiden Ranking como para o Ranking Brasileiro de Pesquisa, o CWTS Leiden considera como possibilidade a auditoria do IREG - que, em 2009, já denominado como IREG Observatory on Academic Ranking and Excellence, ou simplesmente IREG Observatory, criou uma auditoria com o propósito de certificar os rankings que seguem as boas práticas preconizadas pelos Princípios de Berlim e atribui um selo de certificação às listagens auditadas e conformes (IREG, 2009).

BRR Score: Atende (75\%).

\subsection{CATEGORIA: APRESENTAÇÃO DOS RESULTADOS DO RANKING}

\subsubsection{Princípio 15}

O ranking deve proporcionar aos consumidores uma compreensão clara de todos os fatores usados em sua elaboração e oferecer escolhas na forma de apresentação (IREG, 2006).

O BRR é detalhadamente apresentado em seu site oficial, e sua metodologia, como já indicado nos princípios 6 e 7 anteriores, é extensamente explicada pela literatura (Waltman et al., 2011a; 2011b; Waltman et al., 2012; Waltman \& Schreiber, 2013).

Em seu site, o BRR indica as várias formas de proceder à pesquisa das instituições no BRR, e Vogel et al. (2014) mostram pormenorizadamente, por meio de exemplos concretos, como "ler" o BRR. Talvez a dificuldade de entendimento dos resultados do BRR se deva à sofisticação das medidas estatísticas adotadas, o que requer do público interessado a necessária compreensão do significado dos indicadores e como eles são aplicados pelo BRR.

Um ponto importante a ser trabalhado pelo CWTS Leiden refere-se a dispor as informações sobre o BRR em seu site também em português, 
considerando o público falante desse idioma, já que é um ranking focado em instituições brasileiras.

BRR Score: Atende parcialmente (50\%).

\subsubsection{Princípio 16}

Ser compilado de modo a eliminar ou reduzir erros nos dados originais e ser organizado e apresentado de forma tal que erros e falhas possam ser corrigidos (IREG, 2006).

Este princípio é, de certa forma, sobreposto aos princípios relacionados na categoria Metodologias e na categoria Coleta e Tratamento de Dados dos Princípios de Berlim - já cotejados anteriormente com as características do BRR que discutem a fonte de dados utilizada, a metodologia adotada e todos os demais procedimentos voltados à garantia da confiabilidade do ranking, inclusive considerando erros (mais relacionados às inconsistências dos dados extraídos de bases de dados).

BRR Score: Atende plenamente (100\%).

Os scores atribuídos ao BRR de acordo com a sua conformidade a cada um dos 16 Princípios de Berlim são consolidados na Tabela 1.

\section{Tabela 1: Conformidade do BRR aos Princípios de Berlim para rankings de IES}

\begin{tabular}{|c|c|c|c|c|c|c|}
\hline \multicolumn{2}{|c|}{ Princípios de Berlim } & $\begin{array}{l}\frac{\pi}{0} \\
\frac{0}{0} \\
\pi \\
0 \\
0 \\
0 \\
2 \pi \\
2\end{array}$ & 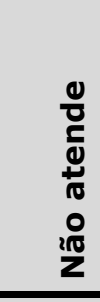 & 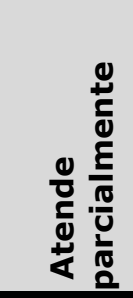 & 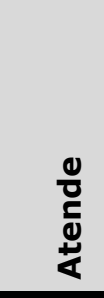 & 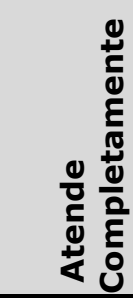 \\
\hline Categoria & Princípio & NA & $0 \%$ & $50 \%$ & $75 \%$ & $100 \%$ \\
\hline \multirow{5}{*}{$\begin{array}{l}\text { Objetivos e } \\
\text { Metas }\end{array}$} & 1 & & & & $\bullet$ & \\
\hline & 2 & & & & • & \\
\hline & 3 & & & & $\bullet$ & \\
\hline & 4 & & & & $\bullet$ & \\
\hline & 5 & & & & $\bullet$ & \\
\hline \multirow{3}{*}{ Metodologia } & 6 & & & & & • \\
\hline & 7 & & & & & $\bullet$ \\
\hline & 8 & & & & & $\bullet$ \\
\hline
\end{tabular}




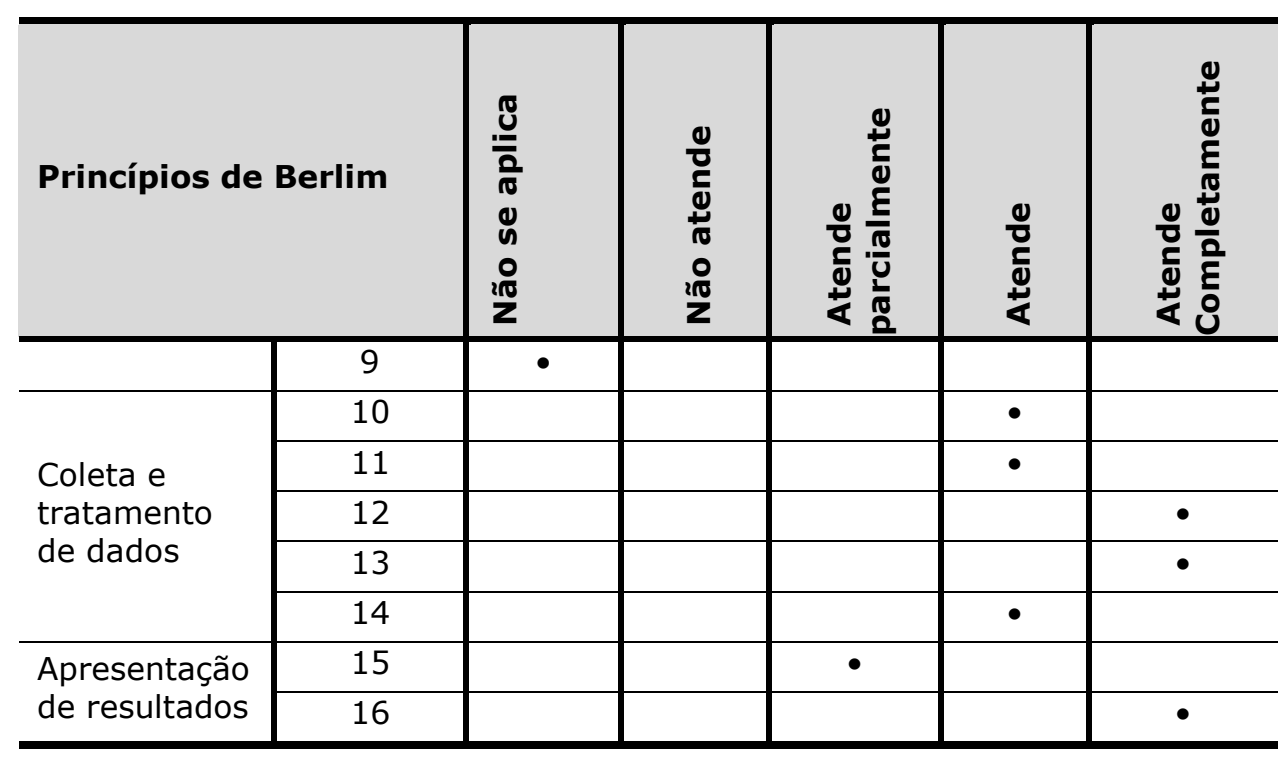

Fonte: elaboração própria

Percebe-se que a conformidade do BRR predomina em "Atende" (com valor de $75 \%$ de conformidade) em oito dos Princípios de Berlim, sendo a maioria na categoria Objetivos e metas dos rankings, em que o BRR está conforme em todos os requisitos; seguido de "Atende completamente", com 100\% de conformidade, em três princípios da categoria Metodologia dos rankings, e o 90 princípio dessa categoria - sobre os pesos atribuídos aos indicadores dos rankings - não se aplica, uma vez que o BRR não adota ponderações em seus indicadores. O BRR também atinge $100 \%$ de conformidade em dois princípios da categoria Coleta e tratamento dos dados; e ainda em um princípio da categoria Apresentação de resultados. O único princípio em que a conformidade do BRR figurou como "Atende parcialmente" refere-se ao $16^{\circ}$ da categoria Apresentação de resultados, devido à percepção da necessidade de o BRR manter também uma interface em português no seu site, que atualmente é somente em inglês. O BRR não figurou em nenhum score "Não atende". Os resultados indicam que o BRR adere à quase totalidade dos Princípios de Berlim para Rankings de Instituições de Ensino Superior, em conformidade com a maioria dos princípios. 


\section{CONSIDERAÇÕES FINAIS}

O recente surgimento dos rankings internacionais monopolizou atenções ao sinalizar padrões de excelência e qualidade do Ensino Superior, causando impacto nos stakeholders - comunidade acadêmica, órgãos gestores de políticas públicas, agências de fomento e mesmo a sociedade, por meio da reputação e expressão da opinião pública, refletida nas escolhas para ingresso no ensino superior. Entretanto, a percepção atribuída aos rankings como fontes incontestáveis de indicação de excelência e qualidade institucional das IES não está livre de controvérsias. Conceitos, dimensões, indicadores, métodos de análise de informações e dados, abrangência, bem como seus efeitos têm sido alvo de amplas discussões.

Nesse sentido, a criação de um conjunto de diretrizes para nortear a elaboração de rankings de forma transparente e ética é uma iniciativa pertinente e bem-vinda, apoiando a melhoria contínua e o aperfeiçoamento das metodologias utilizadas nas diversas classificações.

Neste trabalho, o objetivo foi verificar a adequação do Ranking Brasileiro de Pesquisa (BRR), aos princípios para rankings exortados pelo IREG, uma vez que a sistematização do BRR não é um empreendimento acabado e definitivo, mas, sim, um esforço de melhoria contínua, considerando não só o rigor metodológico aplicado a seus procedimentos, mas também os princípios éticos requeridos e esperados de sistemas de classificação de instituições de ensino superior e de pesquisa, aumentando sua confiabilidade. O cotejo realizado com os Princípios de Berlim para Rankings de Instituições de Ensino Superior mostrou que o BRR apresentase na conformidade "Atende" em oito dos princípios e em "Atende plenamente" em seis dos princípios, indicando que a quase totalidade de suas características está adequada às 16 recomendações indicadas. 


\section{REFERÊNCIAS}

Almeida Filho, N. (2011). Rankings, vikings, masters \& colleges: dilemas da universidade brasileira no contexto da internacionalização. Conferência ministrada no Instituto de Estudos Avançados da Universidade de São Paulo, em 25 de abril de 2011. 16 p. [impresso].

Badri, M. A., Donald, D., \& Donna, D. (1995). A study of measuring the critical factors of quality management. International Journal of Quality \& Reliability Management, 12(20), 36-53.

Buela-Casal, G., Gutiérrez-Martínez, O., Bermúdez-Sánchez, M. P., \& Vadillo-Muñoz, O. (2007). Comparative study of international academic rankings of universities. Scientometrics, 71(3), 349-365.

Cavacini, A. (2015). What is the best database for computer science journal articles?. Scientometrics, 102(3), 2059-2071.

CWTS Brazilian Research Ranking. (2015). Methodology. Leiden: CWTS. Recuperado em 9 de outubro, 2015 de http://brr.cwts.nl/methodology

Folha de S. Paulo (2016). Como é feito o Ranking de. São Paulo: Folha de São Paulo. Recuperado em 25 de setembro, 2016, de http://ruf.folha.uol.com.br/2016/o-ruf/ranking-universidades/

Hood, W. W., \& Wilson, C. S. (2001). The scatter of documents over databases in different subject domains: how many databases are needed? Journal of the American Society for Information Science and Technology, 52(14), 1242-1254.

International Ranking Expert Group - IREG. (2006). Princípios de Berlim para Rankings de Instituições de Ensino Superior. Berlim: IREG. Recuperado em 10 de outubro, 2015, de http://www.iregobservatory.org/index.php?option $=$ com content\&task=view\&id $=41 \&$ Item $\underline{\mathrm{id}=48}$

International Ranking Expert Group - IREG. (2009). IREG ranking audit: purpose, criteria and procedure. Berlim: IREG Observatory. Recuperado em 20 de outubro, 2015, de http://www.unesco.org/new/fileadmin/MULTIMEDIA/HQ/ED/pdf/RANKIN GS/Sadlak IREG.pdf

Lei n. 10.861, de 14 de abril de 2004 (2004). Institui o Sistema Nacional de Avaliação da Educação Superior - Sinaes e dá outras providências. Diário Oficial da União, 72, Seção 1.

Portal de Periódicos da Capes. (2015). Recuperado em 2 de outubro, 2015, de http://www-periodicos-capes-gov-br.ez67.periodicos.capes.gov.br/

Portaria Normativa n. 40, de 12 de dezembro de 2007 (2010, 29 de dezembro). Institui o e-MEC, sistema eletrônico de fluxo de trabalho e gerenciamento de informações relativas aos processos de regulação, 
avaliação e supervisão da educação superior no sistema federal de educação, e o Cadastro e-MEC de Instituições e Cursos Superiores e consolida disposições sobre indicadores de qualidade, banco de avaliadores (Basis) e o Exame Nacional de Desempenho de Estudantes (ENADE) e outras disposições. Diário Oficial da União, 249, Seção 1. [republicação do original de 2007].

Ranking Universitário da Folha (2016). Recuperado em 25 de setembro, 2016, de http://ruf.folha.uol.com.br/2016/o-ruf/ranking-universidades/

Sanz-Casado, E., Garcia-Zorita, C., Serrano Lopez, A. E., Efraín-García, P., \& De Filippo, D. (2013). Rankings nacionales elaborados a partir de múltiples indicadores frente a los de índices sintéticos. Revista Española de Documentación Científica, 36(3), 1-18.

Shin, J. C. (2011). Organizational effectiveness and university rankings. In J. C. Shin, R. K. Toutkoushian, \& U. Teichler. (Eds.), University rankings: theoretical basis, methodology and impacts on global higher education (pp. 19-34). Houten: Springer.

Shin, J. C., Toutkoushian, R. K., \& Teichler, U. (Eds.). (2011). University rankings: theoretical basis, methodology and impacts on global higher education. Houten: Springer.

Shin, J. C., \& Toutkoushian, R. K. (2011). The past, present, and future of university rankings. In J. C. Shin, R. K. Toutkoushian, \& U. Teichler. (Eds.), University rankings: theoretical basis, methodology and impacts on global higher education (pp. 1-16). Houten: Springer.

Thomson Reuters. (2014). Web of Science. Recuperado em 15 de outubro, 2015, de http://wokinfo.com/citationconnection/

Teichler, U. (2011). Social contexts and systemic consequence of university rankings: a meta-analysis of the ranking literature. In J. C. Shin, R. K. Toutkoushian, \& U. Teichler. (Eds.), University rankings: theoretical basis, methodology and impacts on global higher education (pp. 55-69). Houten: Springer.

United Nations Educational, Scientific and Cultural Organization - Unesco. (2015). Unesco Science Report: towards 2030. Paris: Unesco. 795 p. Recuperado em 11 de novembro, 2015, de http://unesdoc.unesco.org/images/0023/002354/235406e.pdf

Usher, A., \& Medow, J. (2009). A global survey of university rankings and league tables. In B. M. Kehm, \& B. Stensaker (Eds.), University rankings, diversity, and the new landscape of higher education (pp. 3-18). Rotterdam: Sense Publishers.

Vogel, M. J. M., Milanez, D. H., Noyons, E., Kobashi, N. Y., \& Faria, L. I. L. (2014). Como ler um ranking: a proposta do Brazilian Research Ranking. In XV Encontro Nacional de Pesquisa em Ciência da Informação (p. 34223438). Belo Horizonte: UFMG. Recuperado em 10 de outubro, 2015, de http://enancib2014.eci.ufmg.br/documentos/anais/anais-gt7 
Waltman, L., van Eck, N. J., van Leeuwen, T. N., Visser, M. S., \& van Raan, A. F. J. (2011a). Towards a new crown indicator: some theoretical considerations. Journal of Informetrics, 5(1), p. 37-47.

Waltman, L., van Eck, N. J., van Leeuwen, T. N., Visser, M. S., \& van Raan, A. F. J. (2011b). Towards a new crown indicator: an empirical analysis. Scientometrics, 87(3), 467-481.

Waltman, L., Calero-Medina, C., Kosten, J., Noyons, E. C. M., Tijssen, R. J.W., et al. (2012). The Leiden Ranking 2011/2012: data collection, indicators, and interpretation. Journal of the American Society for Information Science and Technology, 63(12), 2419-2432.

Waltman, L. \& Schreiber, M. (2013). On the calculation of percentile-based bibliometric indicators. Journal of the American Society for Information Science and Technology, 64(2), 372-379.

Webster, T. J. (2001). A principal component analysis of the US News \& World Report tier rankings of colleges and universities. Economics of Education Review, 20(3), 235-244. 